\title{
SEFLab: A Lab for Measuring Software Energy Footprints
}

\author{
Miguel A. Ferreira*, Eric Hoekstra ${ }^{\dagger}$, Bo Merkus ${ }^{\dagger}$, Bram Visser*† and Joost Visser*‡ \\ * Software Improvement Group \\ Amsterdam, Netherlands \\ Email: \{m.ferreira,b.visser,j.visser $\} @$ sig.eu \\ ${ }^{\dagger}$ CleanTech, Hogeschool van Amsterdam, \\ Amsterdam, Netherlands \\ Email: \{e.hoekstra,b.merkus\}@hva.nl \\ ${ }_{\ddagger}^{\ddagger}$ Radboud University Nijmegen, \\ Nijmegen, Netherlands
}

\begin{abstract}
Hardware dissipates energy because software tells it to. But attributing hardware energy usage to particular software functions is complicated due to distribution, resource sharing, and layering of software. To enable research on energy usage attribution, we have created the Software Energy Footprint Lab. We explain the experimental setup offered by the lab and the measurement and analysis methodology that it supports. We also describe some preliminary results aimed at deciphering hardware dissipation profiles for various types of servers under various forms of software stress. Finally, we provide an outlook of how energy footprint measurements can contribute to a body of knowledge on software-level energy optimization.

Index Terms-Software Engineering, Energy efficiency, Green products
\end{abstract}

\section{INTRODUCTION}

The ICT sector is responsible for substantial and fastgrowing energy consumption [1]. At the level of hardware, datacenters, and energy grid important progress has been made in improving efficiency and reducing energy loss. However, design and development of software applications remains mostly energy-agnostic. With the exception of programming for mobile devices -where battery life is major concernapplication programmers rarely optimize their software for energy consumption. As a result, gains in energy-efficiency in hardware and infrastructure could be cancelled out by inefficient resource consumption of software.

As an important step towards energy-aware software design and development the relationship must be mapped out between software operation and energy dissipation in hardware. This relationship is complicated due to the distribution of software components over multiple hardware devices, the sharing of hardware devices by multiple software components, and the layered design of modern software with frameworks, virtual machines, interpreters, and other abstraction mechanisms.

We have initiated a laboratory to study the energy footprint of software. The setup of the lab includes measurement infrastructure that enables measurement at hardware and software levels and alignment of the resulting measurement streams. To allow accurate measurement, fine-grained server instrumentation has been put in place.
In this paper, we present the setup of our energy footprint lab (Section II) and we share some initial experimental results (Section III). We also discuss related work (Section IV), remaining weaknesses (Section V), and we provide an outlook to further improvement and experimentation (Section VI).

We provide a replication package for this research via the url: http://www.sig.eu/en/seflab-paper.

\section{LAB SETUP}

\section{A. Infrastructure}

Fig. 1 depicts a conceptual view of the SEFLab. Seen as a black-box, the SEFLab takes software products in, executes them in a server and outputs power measurements taken during the software execution.

To understand the measurements produced by the setup, one needs to analyze how it is built. The three main components of the setup are (1) the server (where the software under test is executed), (2) the data acquisition system (DAQ) (that collects all measurements and provides them in a machine readable format) and (3) the measurements PC (where all measurements are processed and recorded).

Fine-grained instrumentation is applied to the server under test. The situation in the figure is based on the server currently under test, which is a Dell PowerEdge SC1425 with the following specifications:

- 2x Intel Xeon CPUs, $3.2 \mathrm{GHz}$

- 4x Infineon 1GB DDR2-333 SDRAM

- Intel E7520 chipset

- 1x Maxtor 7L250S0 250GB SATA150 HDD

- Dell power Supply Unit 450W

This server is rigged with sensor boards (green arrows) attached to the power distribution lines that go from the power supply unit (PSU) to the motherboard. This way we can obtain independent power measurements for several components. (The arrow going from the memory bank to the sensor board is dashed because it was physically impossible for us to isolate the memory power distribution line. Still we were able to calculate the power dissipated in the memory banks via extra 


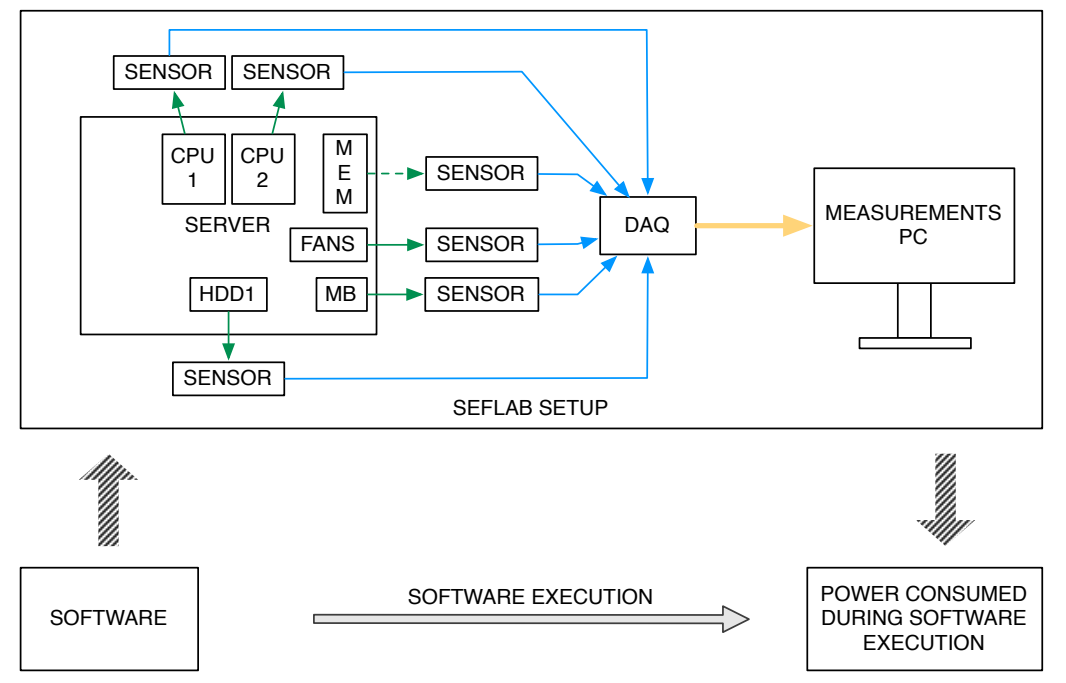

Fig. 1. SEFLab's infrastructure.

calculations. Section IV provides more details on how these calculations are made.) Note that most components themselves have small dedicated PSUs incorporated which regulate the higher voltage from the main PSU back to the component's actual working voltage. We measure the component current and voltage just before these dedicated PSUs. Each sensor board has a sense resistor that enables us to measure the current that flows through it and a filter circuit that filters out undesired high frequency noise from the signals that we want to measure. Both the sense resistor and the filter are selected in accordance with the electrical characteristics of the specific power distribution line. The voltages of the power distribution lines are measured non-intrusively by the setup. Also here a dedicated filter circuitry is applied.

All sensor boards are connected to separate channels of the DAQ (blue arrows). The DAQ samples all the signals coming from the sensor boards, while making the digitalized data available for further processing in the measurements PC (yellow arrow). Hereby a sampling frequency of $30 \mathrm{kHz}$ is chosen to sample at least at a 10 times higher frequency than the cut-off frequencies of the 3rd order filters.

Once the data is received at the measurements PC it is further processed in LabView to, for instance, reduce the volume of data. The measurements PC allows for real-time visualization of the data as well as to store it in file.

One additional piece of the setup that is not depicted in the picture for simplicity, is a USB to serial converter that connects a USB port on the server to an independent channel in the DAQ. This extra connection allows us to send a pulse to the DAQ right before the software under test is executed and another one right after it terminates. These pulses will be converted into timestamps that enable us to align the power measurements gathered in the measurements PC with other types of measurements (possibly gathered elsewhere). One example of such other measurements are the computational resources (CPU, memory etc.) being used in the server while it executes the software under test. Knowing the computational resources being used along side with the power measurements allows for a deeper understanding of what the software is doing and how that relates to power dissipation on the server.

\section{B. Methodology}

Server vendors do not publicly publish information about the design of their products at a level of granularity that would help someone interested in building a setup such as the one found in the SEFLab. Therefore finding which electrical wires coming out of the PSU feed electricity to which server components is a challenge. The methodology used at the SEFLab was inspired in the method used in [2] where other researchers were facing the same difficulties. We connected one multimeter to each electrical wire coming out of the PSU and set it to measure current. Then, having tapped all electrical wires, we used a tool that can stress the different components of the server individually and monitored the response in the multimeters. By doing so we could identify which were the electrical wires feeding each component of the server and therefore could reconstruct the conceptual power distribution network implemented in the many layers of circuits hidden in the motherboard of the server. In addition to knowing which electrical wires feed which components we also learned about their electrical characteristics, the peak current that goes through them. How much current goes through each wire is an important factor for deciding on the resistance of the sense resistor to be installed.

Using this methodology all the electrical wires from the PSU were mapped one-to-one to all components of the server, except for the memory banks and the fans. The memory banks, the case and CPU fans share the same electrical wires. Luckily the fans are connected to the motherboard by yet another set of electrical wires that we could intercept to measure both voltage and current. Knowing the voltage and current that is supplied 
to the fans alone we could deduct those signals from the ones that combine the fans with the memory and reconstruct the signals of the memory alone. This approach to calculate the power dissipated in the memory banks leads to a less accurate value when compared with the others. The exact magnitude of this deviation is left to future development of the SEFLab.

To finalize, the current setup of the SEFLab was built by two Electronics Engineering students from the Hogeschool van Amsterdam (HvA) during their BSc graduation projects [3], [4] and later validated by another student (the fourth author of this paper) also as BSc graduation project [5]. The validation of the SEFLab setup consisted of (1) an investigation of the frequency spectrum of the different components, (2) an investigation of the design of the filters, (3) selecting the sampling frequency according to the NyquistShannon sampling theorem, and (4) to ensure low voltage drop on the measured channels (smaller than 1\%), to not alter the components' behavior.

\section{Preliminary Measurements}

In this section we report on two experiments we ran on the SEFLab. In the first experiment (Section III-A) a stress test tool, named HeavyLoad ${ }^{1}$, is used to create load in the server. The objective is two fold: (1) investigate whether there is an observable relation between load placed on hardware components and their power dissipation (power scalability); (2) investigate whether an application can impact the energy dissipated by a computer in total and in its independent hardware components. In the second experiment (Section III-B) we investigate if the SEFLab enables the comparative analysis of different applications with respect to their energy footprint. To this end, we collect and compare power dissipation measurements of several popular web browsers. With the collection of power dissipation measurements in such a structured way, one can envisage benchmarking applications in terms of their power dissipation (and efficiency when sufficiently similar to each other) in a software energy footprint registry.

\section{A. Stress test}

1) Experimental Design: We have conducted some preliminary measurements using the SEFLab setup (Fig. 1) to validate it. For these tests we installed Windows 7 in the lab's server and used a stress test tool called HeavyLoad. The most natural choice of operating system (OS) would be a server edition, however we plan to study the accuracy of software energy profilers and at the time of this experiment there was only one available that runs on Windows (Joulemeter) and it is limited to Windows 7. The choice for HeavyLoad was based on its free availability on the Internet, its capability to stress multiple computational resources simultaneously and independently, and (also very important) it provides a command line interface which integrates well with the data synchronization mechanism explained in the previous section.

For computational resources collection on the server we used a tool that ships with the OS called Perfmon. Perfmon collected data on CPU, memory and hard drive utilization.

\footnotetext{
${ }^{1}$ http://www.jam-software.com/heavyload
}

TABLE I

STRESS TEST SCENARIOS.

\begin{tabular}{ll}
\hline Scenario & \multicolumn{1}{c}{ Load } \\
\hline IDLE & OS and Perfmon \\
\hline CPU & OS, Perfmon and HeavyLoad (CPU test) \\
\hline MEM & OS, Perfmon and HeavyLoad (Memory test) \\
\hline HDD & OS, Perfmon and HeavyLoad (Hard drive test) \\
\hline ALL & OS, Perfmon and \\
& HeavyLoad (CPU, memory and hard drive tests) \\
\hline
\end{tabular}

To calculate the energy dissipated by the CPU, the memory and the hard drive, during the execution of HeavyLoad, we collected measurements of current and voltage for all those components. Remember that we measured the current $I$ by applying a sense resistor $R$, giving a small voltage $V_{R}$ in the millivolt range that represents the actual current (Ohms law). By dividing $R$ with the measured voltage $V_{R}$ the current $I$ is obtained (2). Multiplying this current with the also measured voltage $V$ one obtains the power $P(1)$, which is the energy dissipated per second.

$$
\begin{array}{r}
P=I \times V \\
I=\frac{R}{V_{R}}
\end{array}
$$

Having the power being drawn in each second one only needs to sum it all up to obtain the dissipated energy.

We defined five load scenarios that we ran three times for half an hour each. Table I lists all five scenarios together with the load for each of them. For each run of each scenario the following steps were taken:

1) Turn the server on,

2) Login to OS,

3) Start Perfmon,

4) Start HeavyLoad,

5) Wait for HeavyLoad to terminate,

6) Collect the Perfmon data and the timestamps of when HeavyLoad started and terminated,

7) Turn the server off.

Although the SEFLab setup is capable of taking more than a $100 \mathrm{~K}$ samples per second, we have set the sampling frequency to $30 \mathrm{~K}$ samples per second. We further reduced the sample rate, in Labview via a filtering technique, to 5 samples per second in order to lower the amount of generated measurement data.

2) Results: The implicit hypothesis when building the SEFLab was that we could relate resource dissipation of a software application to the power dissipation of the computer running it. Fig. 2 and Fig. 3 depict CPU utilization (time) percentage and power dissipated by the CPU, respectively. From the figures it is observable that the power dissipated by the CPU stays constant relative to the load, i.e., while the load is constantly high the power drawn is also constantly high. However, this does not hold for all hardware components. What we observe in our setting is in clear contradiction with 


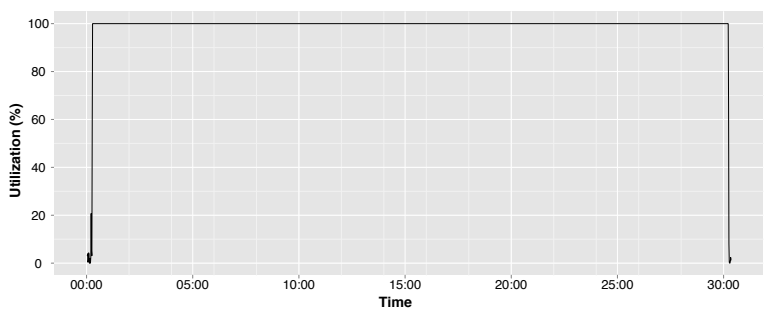

Fig. 2. Percentage CPU utilization for scenario CPU (run 1).

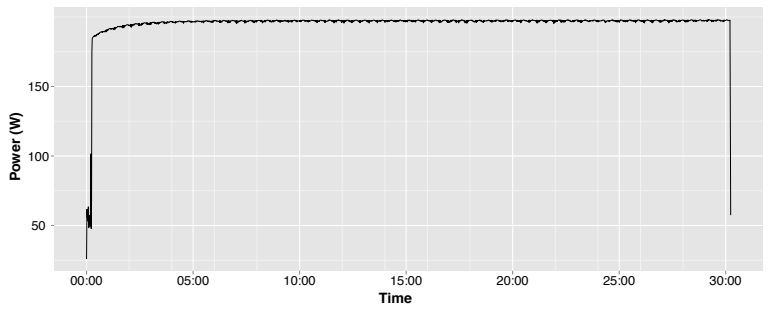

Fig. 3. CPU power dissipation for scenario CPU (run 1).

what other researchers before us observed. The authors of [2] report that, their results "show that CPU utilization is not an accurate reflection of the CPU power". One possible explanation to this apparent contradiction is that the benchmarks used in [2] stress different arithmetic logic units (ALUs) (the parts responsible for calculations) in the CPU (eg. integer arithmetic ALU vs floating point arithmetic ALU). Another possible explanation is that the authors of [2] mean that the power the CPU draws is not completely proportional to its load. Lack of scalability is also reported in [6].

The same comparative analysis for memory shows something completely different. Fig. 4 and Fig. 5 depict the mem-

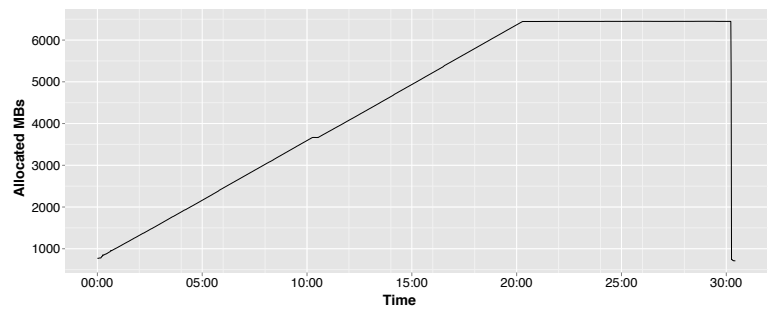

Fig. 4. Allocated Memory in MB for scenario MEM (run 1).

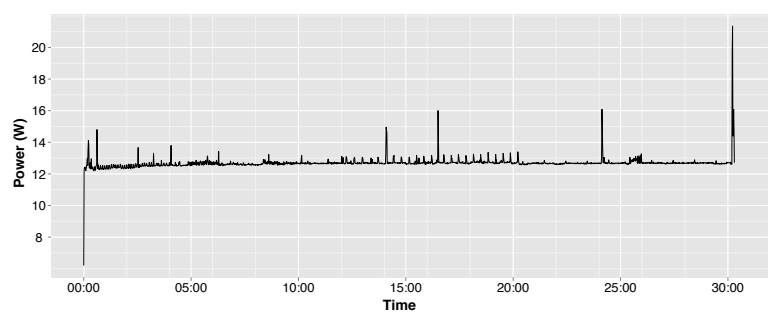

Fig. 5. Memory power dissipation for scenario MEM (run 1). ory utilization (in allocated MBs) and the power dissipated by the memory banks, respectively. From Fig. 4 it is observable that the amount of allocated memory increases linearly during the execution of the scenario, until it hits the (defined) maximum amount. While, Fig. 5 shows that, with the exception of a few spikes, the power dissipation of the memory banks is practically constant during the execution of the scenario. This means that the power dissipation of the memory banks does not change significantly because the application was using more memory. Our analysis here does not include phenomena like memory swapping (writing/reading memory pages to/from disk to to provide applications with more memory than the available amount in hardware). To avoid swapping we defined the maximum amount of memory 1GB bellow the total amount of memory available. As for the hard drives there seems to

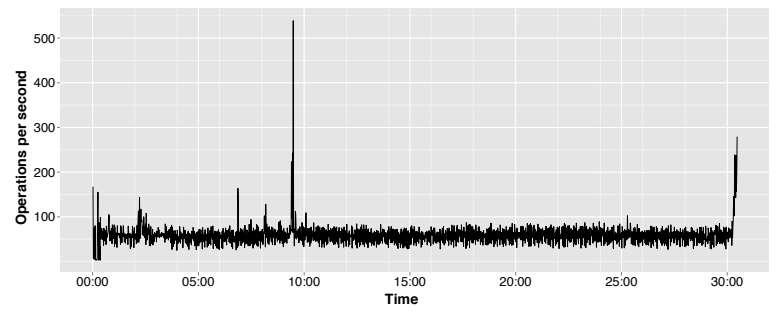

Fig. 6. Hard drive transactions per second for scenario HDD (run 1).

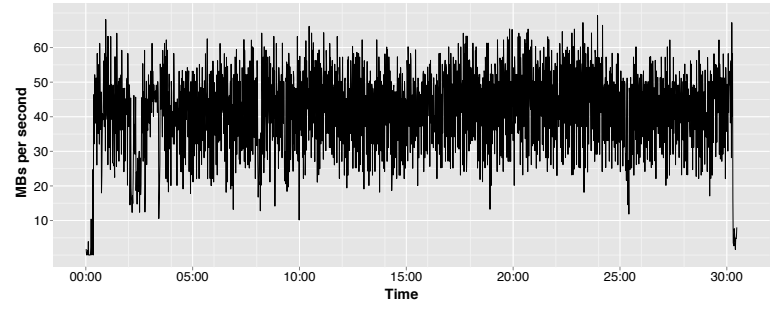

Fig. 7. Hard drive data transfer in MB per second for scenario HDD (run 1).

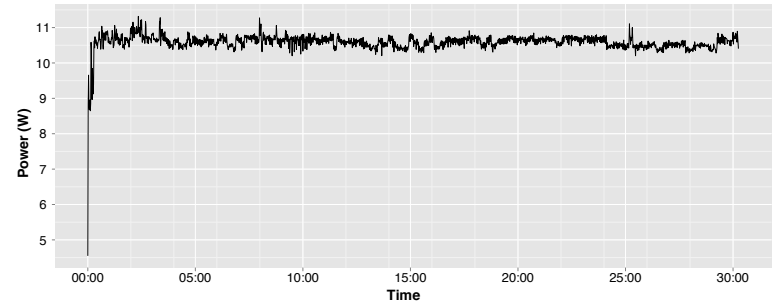

Fig. 8. Hard drive power dissipation for scenario HDD (run 1).

be a similar effect on the power dissipation related to the load as was observed for the CPU. Fig. 6, Fig. 7 and Fig. 8 depict the number of hard drive transactions per second, the data transferred from and to the hard drive in MBs per second and the power dissipated by the hard drive, respectively. Both Fig. 6 and Fig. 7 show a (almost) constant rate of activity in the hard drive, which is matched by the also (almost) constant 
TABLE II

POWER DISSIPATION (IN W) PER RUN FOR EACH SCENARIO.

\begin{tabular}{lrrrrr}
\hline & Run 1 & Run 2 & Run 3 & Average & $\begin{array}{r}\text { Difference } \\
\text { to IDLE }\end{array}$ \\
\hline IDLE & 121.17 & 123.65 & 123.02 & 122.61 & $0(0 \%)$ \\
\hline CPU & 268.42 & 267.20 & 268.41 & 268.01 & $145.40(54 \%)$ \\
\hline MEM & 127.67 & 127.80 & 127.91 & 127.79 & $5.18(4 \%)$ \\
\hline HDD & 154.80 & 156.05 & 155.78 & 155.55 & $32.93(21 \%)$ \\
\hline ALL & 272.67 & 272.77 & 272.05 & 272.50 & $149.88(55 \%)$ \\
\hline
\end{tabular}

TABLE III

POWER DISSIPATION (IN W) PER COMPONENT FOR IDLE AND ALL.

\begin{tabular}{lccr}
\hline & IDLE & ALL & Difference \\
\hline CPU & 48.90 & 193.60 & $144.7(75 \%)$ \\
\hline MEM & 11.98 & 16.21 & $4.23(26 \%)$ \\
\hline HDD & 8.54 & 10.66 & $2.12(20 \%)$ \\
\hline
\end{tabular}

rate of power dissipation shown in Fig. 8. The big difference, from the perspective of the relation load to power dissipation, between the CPU and the hard drive is in the magnitude of the increase in the power dissipation. In the following paragraphs we investigate the magnitude of changes in power dissipation of the computer while executing the HeavyLoad software and quantify the differences by hardware component and in total.

Table II shows total power dissipation for each run of each scenario, as well as the average power dissipation per scenario. The first observation we make is that the application used in this experiment (HeavyLoad) has a very significant impact in the total power dissipation of the server. Comparing the average power dissipation of the IDLE scenario to the average power dissipation of the remaining scenarios we observe that executing HeavyLoad can increase the power dissipation of the server by $4 \%$ in the case where only the memory is stressed, up to $55 \%$ in the case where all three hardware components are stressed. The second observation is that the power dissipation is quite stable between the different runs.

Table II shows total power dissipation per run for each scenario. If the total power dissipation is broken down into the different hardware components it is possible to quantify the contribution of each component in the total power dissipation increase. Table III shows the average power dissipation for the IDLE and ALL scenarios broken down into CPU, memory and hard drive. CPU is by far the hardware component for which there is a bigger increase in power dissipation. In addition, $\mathrm{CPU}$ is also the hardware component (from the components we analyze in this experiment) that dissipates the most power in absolute terms. The increase in the total power dissipation from the IDLE to the ALL scenario is $149.88 \mathrm{~W}$ (Table II). The increase in the CPU power dissipation from the IDLE to the ALL scenario is $144.7 \mathrm{~W}$ (Table III). This means that the power dissipation of the CPU accounts for $97 \%$ of the total power dissipation increase. These findings are inline with findings of [7] which states that "the total system power is still dominated by CPU power in the case of $C P U$
TABLE IV

BROWSER TEST SCENARIOS.

\begin{tabular}{ll}
\hline Scenario & \multicolumn{1}{c}{ Load } \\
\hline IDLE & OS \\
\hline GOOGLE.NL & OS and browser (loading www.google.nl) \\
\hline GOOGLE.COM & OS and browser (loading www.google.com) \\
\hline YOUTUBE & OS and browser (loading www.youtube.com) \\
\hline LIVE & OS and browser (loading www.live.com) \\
\hline LINKEDIN & OS and browser (loading www.linkedin.com) \\
\hline WIKIPEDIA & OS and browser (loading www.wikipedia.org) \\
\hline NU & OS and browser (loading www.nu.nl) \\
\hline YAHOO & OS and browser (loading www.yahoo.com) \\
\hline MARKTPLAATS & OS and browser (loading www.marktplaats.nl) \\
\hline
\end{tabular}

intensive workloads". On the other hand, our preliminary results contradict the report from [8] where it is said that: "(... ) memory power consumption is likely to be equally, if not more, important [to CPU] in the future (...)".

\section{B. Browser comparison}

1) Experimental Design: The objective of this experiment is to investigate how to compare similar applications on their power dissipation properties. The same setup was used as described in Section III-A1 with the exception of the sampling frequency which was set to $50 \mathrm{~Hz}$ (as opposed to $5 \mathrm{~Hz}$ in the previous experiment). In this test we report results in units of power and energy. Power is the rate of energy dissipation per second, therefore having the power dissipation measurements (in watt - W) one only needs to multiply that value by the duration (in seconds) to obtain the energy dissipation (in joule - J). The five most commonly used Internet browsers were compared by loading nine of the most visited websites in the Netherlands (see http://www.alexa.com). Internet browsers were chosen because they are widely known and used, they are freely available, Computational resources were not analyzed during this test.

We defined ten load scenarios, combined in one browsing session, which we ran ten times for 15 seconds each. Table IV shows all ten scenarios together with the load for each of them. These ten scenarios were executed ten times each, using a windows batch file. The browsers used in the experiment are Firefox 18, Internet Explorer 9, Opera 12, Safari 5 and Chrome 24. Before each browsing session all cache, cookies and other temporary files were deleted. The following steps were taken to test each browser:

1) Turn the server on;

2) Login to OS;

3) Delete temporary files and execute all scenarios; $(\times 10)$

4) Collect the timestamps of when each scenario started and terminated;

5) Turn the server off.

2) Results: Comparing applications on power dissipation requires measuring the difference to an idle system. Therefore, when a website is loaded, changes in power dissipation on 
the various hardware components are expected. Fig. 9 depicts the power dissipation on the CPUs when the NU scenario is executed. It is visible in the figure that there are significant variations in power dissipation as the scenario is executed.

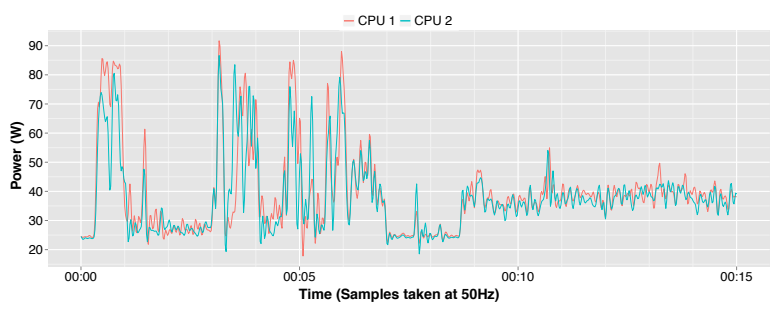

Fig. 9. Average CPU power dissipation of Chrome executing scenario NU.

The mean power dissipation of each hardware component was calculated per scenario by averaging the power dissipation over all ten executions of each scenario. As can be seen in Fig. 10 the power dissipation only slightly increases when loading one of ten websites with Chrome compared to the IDLE scenario. Most power is dissipated in the CPU (as already observed in the experiment of Section III-A), followed by the motherboard and fans. We have calculated the average power dissipation of the combined motherboard and hard drive over all scenarios and found it to be $52 \mathrm{~W}$, the equivalent to $29.5 \%$ of the total consumption. These results seem to be inline with the findings of [8], when it is said that: "more than $30-40 \%$ of the power is spent on the disk, the network, the I/O and peripherals, the power supplies, the regulators, and the rest of the glue circuitry in the server".

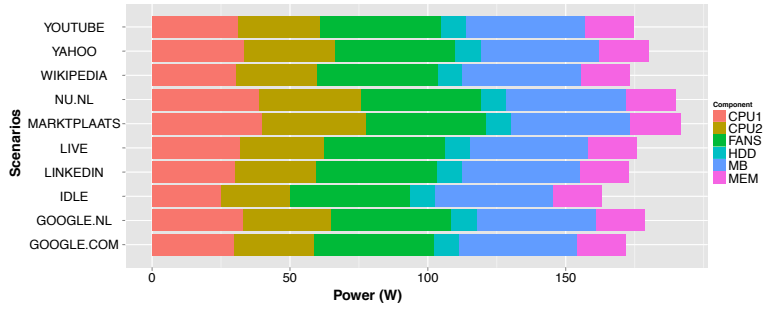

Fig. 10. Average power dissipation of Chrome for all scenarios.

The baseline for comparison of the different scenarios is the IDLE scenario. Fig. 11 depicts the power dissipation of the browsing scenarios executed using Chrome compared to the IDLE scenario. Interestingly, the most power intensive scenario (MARKTPLAATS) adds 29W (18\%) when compared to the IDLE scenario and the least power intensive scenario (GOOGLE.COM) adds 9.1W (6\%).

Table $\mathrm{V}$ shows power and energy dissipation per scenario for each browser. The energy dissipation is what we call the energy footprint. The average energy dissipation per browser varies widely per scenario. For example, Internet Explorer consumes $77 \mathrm{~J}$ to execute the WIKIPEDIA scenario whereas it consumes $560 \mathrm{~J}$ (more than a $7 \times$ increase) to execute the MARKTPLAATS scenario. Opera, on the contrary, consumes

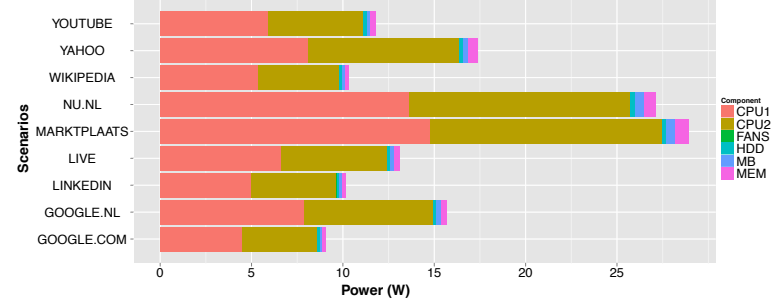

Fig. 11. Average additional power dissipation of Chrome for all scenarios when compared to scenario IDLE.

$205 \mathrm{~J}$ to execute the WIKIPEDIA scenario and $272 \mathrm{~J}$ (not even a $1.5 \times$ increase) to execute the MARKTPLAATS scenario. Analyzing energy dissipation for the five browsers, averaged over the ten runs, shows that website content (i.e. the scenario) significantly impacts the browser energy dissipation. This phenomenon is observable when comparing the power dissipation of MARKTPLAATS and GOOGLE.COM. In the MARKTPLAATS scenario the browsers dissipate more than three times more energy than in the GOOLGLE.COM scenario. This difference is likely to be attributed to the flash content, such as advertisements, loaded in the scenarios MARKTPLAATS and NU, as opposed to the more text based content loaded in the GOOGLE.COM and WIKIPEDIA scenarios. The impact of flash in the power dissipation of browsers has been reported in previous research [9], [10].

We observe a significant difference in the energy footprint of most energy dissipating browser (Opera, with an average of $2288 \mathrm{~J}$ per browsing session) dissipates $69 \%$ more energy than the least energy dissipating browser (Safari, with an average of $1353 \mathrm{~J}$ per browsing session). Furthermore, analyzing browser energy footprints for different scenarios results in different browser rankings. This is an indication that the differences in the implementation of the browsers internal components (e.g. rendering engine, JavaScript engine etc.), despite offering almost the same functionality, have different energy dissipation characteristics. This observation is in line with what is reported in [11] where the different consecutive releases of the same browser show significant differences in energy dissipation.

\section{RELATED WORK}

There are other initiatives similar to the SEFLab. In fact, the SEFLab setup was inspired by the research of [2]. Their research focused on rigging two PCs with digital multimeters tapping into the electrical wires coming from the PSU to the motherboard. Because some components have dedicated electrical wires it is easy to directly measure the power they draw. However, some other components are powered from the motherboard, which in turn is powered by multiple electrical wires. The authors of [2] defined an indirect measurement methodology to account for hardware components that are not powered by dedicated electrical wires. We have, in the SEFLab, partially applied the same methodology to identify the which components are powered by shared electrical wires. In our case the two main chips of the motherboard (North and 
TABLE V

AVERAGE ADDITIONAL ENERGY DISSIPATION (IN JOULE) COMPARED TO SCENARIO IDLE.

\begin{tabular}{lcccccc}
\hline Scenario & Safari & Chrome & Firefox & IE & Opera & Average \\
\hline GOOGLE.NL & 107 & 237 & 111 & 95 & 204 & 151 \\
\hline GOOGLE.COM & 95 & 137 & 99 & 88 & 198 & 123 \\
\hline YOUTUBE & 115 & 177 & 320 & 150 & 230 & 199 \\
\hline LIVE & 191 & 198 & 115 & 93 & 243 & 168 \\
\hline LINKEDIN & 112 & 154 & 97 & 102 & 186 & 130 \\
\hline WIKIPEDIA & 124 & 156 & 169 & 77 & 205 & 146 \\
\hline NU.NL & 217 & 408 & 341 & 330 & 452 & 350 \\
\hline YAHOO & 214 & 261 & 208 & 174 & 298 & 231 \\
\hline MARKTPLAATS & 178 & 436 & 471 & 560 & 272 & 383 \\
\hline TOTAL & 1353 & 2164 & 1931 & 1671 & 2288 & 1881 \\
\hline
\end{tabular}

Southbridge) together with the remaining chips on the board, the network interface cards (NICs) and the graphic card share the same electrical wire (referred to as the motherboard - MB - component). Furthermore the memory banks and all the fans also share one electrical wire. We did not isolate the power consumption of the memory banks using the methodology of [2]. See Section II-B for more details on how the power dissipated in the memory banks is calculated.

Another paper that reports on a similar experiment is [7]. In this paper the power dissipation at hardware component level was measured in a laptop using an oscilloscope and current probes. The same type of subtractive method used in [2] is used in [7] to quantify power dissipation in hardware components that are not directly measurable.

Experiment wise, our results seem to contradict the results of [2] and corroborate the results of [7]. However, further research is required to investigate whether there are in fact contradictions in these results and if so, what is causing it.

In [8] the authors introduce a non-intrusive method, named Mantis, for modeling full-system power dissipation with the capability to predict power dissipation in real-time. In the development of Mantis, the authors studied the componentlevel power dissipation of a blade server. Their approach to study component-level power dissipation also involved rigging the hardware with measurement circuitry. Their report is, however, contradictory to some of our observations (see Section III-A2 for more details).

In [11] the author investigated the power dissipation at the socket level (the entire hardware combined) with changes to software. His results show significative differences between releases of Firefox browser and the Azureus bittorrent client. Comparing to the measurements of the SEFLab there is a great difference in granularity. In the SEFLab we measure power dissipation at hardware component level enabling a better root cause analysis of observed differences. For example, Table III states clearly that the power increase from scenario IDLE to ALL is induced by the CPU. In a similar setup as we describe,
Microsoft researchers argue ${ }^{2}$ that Internet Explorer is the most energy efficient browser. This contradicts our findings. In our preliminary results, Safari was the most energy efficient browser. This difference could be explained by differences in the hardware used (laptop vs. servers), as well as differences in software versions and installed plugins. Another explanation for the different results could be the differences in the test scenarios. This raises the question of what would be the ideal unit of work for browsers.

\section{THREATS TO VALIDITY}

We identify threats to validity in our study, amongst the most relevant is a lack of variety in our experimental setup. Currently we measure in one server of a given brand and model running one OS. This hinders the generalizability of our observations beyond the server, the OS or the application under test. However, the preliminary tests already raised questions regarding results of previous research. We acknowledge the need to broaden our research and we intend to do so.

Another relevant threat to validity has to do with the reliability and accuracy of the measurements obtained in the SEFLab. We have gone to great lengths in validating the SEFLab setup. We have followed a standard methodology based on the most fundamental laws of electronics to designs the circuits we built and integrated in the server. Non the less, the graduation assignment of the fourth author of this paper was to investigate possible measurements errors in the SEFLab setup. From this validation project we concluded that the measurements taken with the SEFLab setup have an error margin close to $1 \%$, which seems acceptable to us. We are busy with a new validation method that will bring that error margin bellow $1 \%$. Furthermore, we will improve the SEFLab setup to cater better for higher frequency ranges, dealing with electromagnetic compatibility and other high frequency effects.

The total energy dissipation measured in our setup is the sum of the energy flowing from the PSU to the motherboard.

\footnotetext{
${ }^{2}$ http://blogs.msdn.com/b/ie/archive/2011/03/28/ browser-power-consumption-leading-the-industry-with-internet-explorer- 9 . aspx
} 
However, the efficiency of the PSU (approximately $70 \%{ }^{3}$ ) is not taken into account in this research. A WattupPro measuring device will be used in future SEFLab research to measure between the wall socket and the PSU.

\section{CONCLUSIONS}

In this paper we presented the SEFLab measurement infrastructure and examples of how and what it can be used for.

\section{A. Discussion}

With a setup such as the one of SEFLab it is possible to take accurate measurements of power dissipation at the level of independent hardware components (CPU, memory, hard drives etc.). With such power measurements, researchers can start to analyze the relation between the computational resources software engineers work with, and the power dissipation of the underlying hardware. This relation is paramount to provide actionable recommendations to software engineers, because in the development process they reason about abstract computational resources as opposed to the concrete hardware that provides those computational resources.

In fact this separation between the software and the hardware is desirable and has led to immense advances in software engineering. Just imagine were would we stand now if programmers would still be tangled in programming for specific hardware platforms and dealing with all sorts of low-level mechanisms. We came a long way since those days but, as a drawback, ended up with a deceiving notion of unlimited resources that can be spent at will.

Now we have to take a step back, but not so far as to say that we need to start programming the hardware directly again. We have to be able to relate computational resources to power dissipation and that includes how many resources are dissipated but, probably, also how are they used (eg. continuously or intermittently), for how long, for what purpose, etc. This is, in our opinion, what software engineers need, to reason about the power dissipation of applications without having to compromise the abstraction power of programing languages, frameworks, compilers and hypervisors.

\section{B. Contributions}

This paper shows that the relation between power dissipation and computational resources at the component (CPU, memory, etc) level is observable and that it seems to correlate. Furthermore, from our preliminary results, it is observable that power dissipation related to load does not present the same characteristics for all hardware components. For CPU and hard drive there seems to be a correlation between the load they are under and the energy they dissipate. However for the hard drive, the change in power dissipation when under stress is practically negligible. Whereas for the CPU the power dissipation can increase up to 55\%. On the other hand, for memory it seems that the load does not impact the energy

\footnotetext{
${ }^{3}$ http://www.energystar.gov/ia/partners/product_specs/program_reqs/ computer_server_prog_req.pdf
}

dissipation. This means that, in the SEFLab's server, using more memory does not imply more energy dissipation.

The browsers experiment showed that the SEFLab setup caters for comparing applications on energy dissipation. Furthermore, we found significant differences in the energy dissipation of browsers, with the most energy intensive browser dissipating $69 \%$ more energy than the least energy intensive browser.

\section{Future work}

The work presented in this paper lays the ground work for further experimentation. Ultimately, we will aim to create a repository of softare engergy footprints as a body of knowledge to help steer software engineers in the direction of more energy efficient software. In addition such a repository may serve as a benchmark against which software energy efficiency could be certified as many consumer goods are nowadays.

\section{ACKNOWLEDGMENT}

The authors would like to thank Vincent Tseng and Marco van Veen who actively participated in the preliminary research and construction of the SEFLab infrastructure. The authors would also like to acknowledge the vital contribution of Michiel Cuijpers, Robert van den Hoed and Ilja Heitlager without whom the SEFLab would not have been possible to build. Finally, the authors would like to thank the Software Improvement Group, the SURF Foundation and Shuberg Philis for their financial support and guidance.

\section{REFERENCES}

[1] EPA, "EPA Report to Congress on Server and Data Center Energy Efficiency," U.S. Environmental Protection Agency, Tech. Rep., 2007.

[2] H. Chen, S. Wang, and W. Shi, "Where does the power go in a computer system: Experimental analysis and implications," in IGCC. IEEE Computer Society, 2011, pp. 1-6.

[3] V. Tseng, "Software energy footprint lab: Measuring the power consumption in a server at component level," Software Improvement Group and Hogeschool van Amsterdam, BSc Dissertation, October 2012.

[4] M. van Veen, "Software energy footprint lab," Software Improvement Group and Hogeschool van Amsterdam, BSc Dissertation, September 2012.

[5] B. Visser, "Software energy footprint lab: A proven view of the furture," Software Improvement Group and Hogeschool van Amsterdam, BSc Dissertation, February 2013.

[6] L. A. Barroso and U. Hölzle, "The case for energy-proportional computing," IEEE Computer, vol. 40, no. 12, pp. 33-37, 2007.

[7] A. Mahesri and V. Vardhan, "Power consumption breakdown on a modern laptop," in PACS, ser. Lecture Notes in Computer Science, B. Falsafi and T. N. Vijaykumar, Eds., vol. 3471. Springer, 2004, pp. $165-180$.

[8] D. Economou, S. Rivoire, C. Kozyrakis, and P. Ranganathan, "Fullsystem power analysis and modeling for server environments," in MoBS, June 2006, pp. 70-77.

[9] T. Kim, Y. Lee, and Y. Lee, "Energy measurement of web service," in e-Energy, M. A. Marsan, S. Goyal, S. Xu, A. Fernández, M. Prodanovic, and K. Christensen, Eds. ACM, 2012, p. 27.

[10] N. Thiagarajan, G. Aggarwal, A. Nicoara, D. Boneh, and J. P. Singh, "Who killed my battery?: analyzing mobile browser energy consumption," in $W W W$, A. Mille, F. L. Gandon, J. Misselis, M. Rabinovich, and S. Staab, Eds. ACM, 2012, pp. 41-50.

[11] A. Hindle, "Green mining: A methodology of relating software change to power consumption," in MSR, M. Lanza, M. D. Penta, and T. Xi, Eds. IEEE, 2012, pp. 78-87. 